\title{
Tradurre l'orientamento in pratica: laboratori per lo sviluppo delle prefigurazioni professionali degli studenti e delle studentesse
}

\author{
di Francesca Bianchi, Nicolina Bosco^, Caterina Garofano \\ Alessandra Romano ${ }^{\circ *}$
}

\begin{abstract}
Riassunto
Il presente contributo si inserisce all'interno del Progetto "SUPER - Percorsi di orientamento e tutorato per promuovere il successo universitario e professionale" promosso dal Dipartimento di Scienze della formazione, scienze umane e della comunicazione interculturale dell'Università di Siena. L'articolo descrive i laboratori e gli interventi di tutoring realizzati con studenti e studentesse del Corso L-19 dell'Ateneo senese e con studenti delle scuole secondarie di secondo grado del territorio aretino al fine di promuovere percorsi di orientamento al lavoro che favoriscano la scelta consapevole del percorso accademico supportando lo studente nella costruzione della propria traiettoria professionale. Le due esperienze di percorsi laboratoriali presentati in questa sede sottolineano la necessità dell'uso delle metodologie riflessive per intercettare nuovi schemi di intervento ed azione per l'orientamento universitario.
\end{abstract}

Parole chiave: orientamento, giovani, transizione, professione

Abstract. Translating guidance into practice: workshops for the development of professional prefigurations of students

This contribution is a part of the Project entitled "SUPER - Guidance and tutoring paths to promote the university and professional success" promoted by the Department of Education, Human Sciences and Intercultural Communication of the University of Siena. The article describes the

\footnotetext{
${ }^{\circ}$ Università degli Studi di Siena.

^Università degli Studi di Siena. Corresponding author: francesca.bianchi@unisi.it.

* Il contributo è frutto del lavoro comune delle autrici. Solo per ragioni di attribuzioni scientifica, si specifica che Francesca Bianchi è autrice di Sviluppare l'orientamento al lavoro come strumento di transizione alla professione, Nicolina Bosco di Domani è già qui: orientamento al lavoro e future studies, Alessandra Romano di Costruire prefigurazioni professionali all'università. I laboratori di Imprenditoria, genere e leadership femminile, Caterina Garofano di Professioni da scoprire: i percorsi laboratoriali nella scuola secondaria.
}

Educational Reflective Practices (ISSNe 2279-9605), 2/2021

Doi: 10.3280/erp2-2021oa12112 
workshop and tutoring interventions carried out with students of the Bachelor degree (L-19, University of Siena) and high school students to promote career guidance paths that favour an aware choice of the academic path supporting students in building their professional trajectory. The two workshops presented here underline the need to use reflective methodologies in order to intercept new trajectories of intervention and effective actions for the university guidance.

Keywords: guidance, young people, school to work transition, career

First submission: 07/12/2020, accepted: 30/01/2021

Available online: 02/07/2021

\section{Sviluppare l'orientamento al lavoro come strumento di transi- zione alla professione}

Perché l'orientamento al lavoro e alla professione è diventato ancora più importante di ieri? Perché le immagini del lavoro che ci portiamo dietro, e che tendiamo a vedere con nostalgia come tutte le cose d'una volta, purtroppo non reggono il confronto con i cambiamenti in atto. Anziché rimpiangere quel che ci lasciamo dietro, dobbiamo cercare di capire come e perché $i$ soggetti e i contesti del lavoro evolvono, diversificandosi ben oltre le immagini ereditate dal Novecento. Del resto, passare dal mondo del Lavoro maiuscolo fin qui conosciuto a un universo di lavori comporta delle traversie, ma offre anche delle opportunità (Accornero, 1999, p.132).

La citazione del compianto Aris Accornero stupisce per capacità di diagnosi rispetto ad uno scenario oggi piuttosto evidente. Già alla fine degli anni '90 il sociologo del lavoro metteva in guardia rispetto ad una visione dell'orientamento poco in linea con i tempi. Rispetto al paradigma taylorfordista che aveva retto il '900 (il secolo del Lavoro), agli inizi degli anni 2000 lo scenario appariva profondamente mutato. Risultava ad esempio evidente lo sviluppo, in campo educativo e lavorativo, di traiettorie biografiche giovanili molto meno lineari e in minore sintonia (e continuità) rispetto alle carriere genitoriali. I livelli di istruzione sono via via cresciuti e, seppure l'Italia continui ancora oggi a mostrarsi un paese dove i destini dei figli sono profondamente legati a quelli familiari, è indubbio che i giovani abbiano iniziato a scegliere l'attività lavorativa con un grado maggiore di libertà e autonomia. 
Nel nostro paese il ruolo delle istituzioni e dei corpi intermedi si è progressivamente ridotto ed è anche per questo motivo che l'orientamento ha assunto un ruolo essenziale perché le opportunità di scelta individuale hanno via via implicato la necessità di capire meglio come muoversi e quindi essere più capaci di orientarsi. L'orientamento, inoltre, ha acquisito una maggiore rilevanza in una società quale quella italiana caratterizzata da una tradizionale separatezza tra educazione e mondo del lavoro. Da noi la transizione scuola-lavoro è stata tradizionalmente molto più lunga, tortuosa e complessa rispetto ad altri contesti: se il periodo delle scelte si è allungato, l'importanza e la consistenza dell'azione di orientamento non potevano che aumentare di pari passo (Besozzi, 1998; Bianchi, 2005).

Sempre Accornero (1999) ricordava come nel mondo del lavoro tendesse a diminuire il numero dei mestieri compatti e duraturi perché costruiti da un solido insieme di conoscenze e competenze o perché sviluppati da ulteriori specializzazioni; l'eventualità di un declino o di uno spiazzamento professionale diventava più incombente rendendo indispensabile la formazione continua: passando da un lavoro all'altro l'individuo avrebbe dovuto imparare a destreggiarsi tra lavori diversi mentre a meno lavoratori rispetto al passato, sarebbe capitato di svolgere lo stesso mestiere per tutta la vita: anche per tali motivi l'orientamento sarebbe diventato uno snodo insostituibile tra soggetti e contesto.

Accanto ai mutamenti nella sfera educativa e professionale, va ricordato l'aspetto normativo. Negli ultimi quarant'anni, i sistemi universitari europei hanno conosciuto cambiamenti strutturali relativamente a politiche, governance, offerta formativa, numerosità e composizione della popolazione studentesca, finalità dell'istruzione superiore (Vaira, 2007). Le riforme degli ordinamenti, ad esempio, hanno previsto un ampliamento dei compiti del docente universitario che, accanto alle funzioni di ricerca e didattica, hanno presupposto l'impegno nelle attività di orientamento, tutorato come anche nella formazione integrativa degli studenti (Moscati, 2004). Si è progressivamente affermato il principio del riconoscimento della centralità degli studenti e, in quest'ottica, l'orientamento è stato concepito come un processo formativo continuo avente come obiettivo il potenziamento nei giovani delle capacità utili ad auto-orientarsi, partecipando attivamente agli ambienti di studio e di lavoro. Anche l'Unione Europea ha affidato all'orientamento il ruolo di connettore tra $\mathrm{i}$ bisogni dell'individuo e la società secondo percorsi sempre più articolati di formazione-lavoro-formazione nell'arco dell'intera vita professionale ${ }^{1}$ (Messeri, 2004). Si è così fatta strada l'esigenza di assicurare un'attività di orientamento sistemica e coerente, incrementando la

\footnotetext{
${ }^{1}$ La strategia di «Lisbona 2010», «Europa 2020» e la «comunicazione di Bruges» (2010)
} 
motivazione dello studente rispetto alla scelta accademica, aiutandolo a definire obiettivi chiari, rendendolo attivo e partecipe rispetto alla vita universitaria, rafforzandone le competenze trasversali utili per il percorso di studio e il supporto dell'intera carriera. In questa direzione, il docente universitario è diventato una figura chiave, un docente-tutor nel suo ruolo di supporto nell'acquisizione di competenze relative al 'conoscere' (sapere), al 'fare' (abilità e capacità) e 'all'essere' (attitudini e responsabilità). Ė qualcuno che da un lato favorisce l'adattamento e facilita lo sviluppo di un processo formativo atto a promuovere l'apprendimento autonomo in un'ottica personale (lo studente come "persona" quindi le aspettative, le abilità, gli interessi, ecc.) e accademica (l'organizzazione degli studi, il rendimento); dall'altro, tende a sviluppare la sua professionalità per la transizione alla vita professionale.

Se l'orientamento è divenuto un "valore permanente nella vita di ogni persona" accompagnando l'intero percorso degli studi (MIUR, 2014), svolge un ruolo essenziale anche dal punto di vista dell'orientamento in uscita e quindi della prefigurazione degli scenari lavorativo-professionali da parte dello studente (Da Re \& Riva, 2018). L'orientamento è lo strumento attraverso il quale gli studenti possono effettuare scelte motivate, in linea con le proprie aspirazioni in modo da evitare rischi di dispersione iniziale (mancato conseguimento di crediti formativi) e/o abbandono degli studi (dopo il primo anno di università): in effetti, è grazie a scelte oculate che gli studenti possono realizzare una carriera universitaria positiva (Rossi, 2017), ma diventa anche strategico per mettere a punto la propria traiettoria professionale. È sulla base di tali considerazioni che 1'Università di Siena ha promosso un progetto di orientamento al lavoro che ha visto la compartecipazione di un nutrito gruppo di attori composto da docenti e studenti universitari insieme a docenti e studenti delle scuole superiori. Il progetto "SUPER - Percorsi di orientamento e tutorato per promuovere il successo universitario e professionale" promosso dal Dipartimento di Scienze della formazione, scienze umane e della comunicazione interculturale dell'Università di Siena, in collaborazione con 14 atenei del territorio nazionale, ha proposto: a) laboratori e interventi di tutoring da parte dei docenti universitari e di peer tutoring da parte degli studenti dello stesso percorso universitario, utili a supportare gli/le studenti dei tre anni dei corsi L-19, Scienze dell'educazione e della formazione; b) laboratori realizzati con gli/le studenti del quarto e quinto anno delle scuole superiori dei diversi territori coinvolti. I laboratori di tutoring e peer tutoring per gli/le studenti dei corsi di laurea L-19 hanno avuto

hanno definito le linee di azione per l'orientamento efficaci per le istituzioni scolastiche e universitarie. 
l'obiettivo di rinforzare l'empowerment degli studenti universitari non solo dal punto di vista del percorso accademico ma soprattutto ai fini dello sviluppo delle prefigurazioni professionali. I laboratori con gli/le studenti delle scuole superiori hanno avuto, invece, l'obiettivo di sfatare gli stereotipi relativi alla professionalità dell'educatore professionale socio-pedagogico.

\section{Domani è già qui: orientamento al lavoro e future studies}

La prefigurazione del futuro professionale richiama in sé la dimensione educativa, i setting dell'apprendimento e gli spazi formali e informali in cui sperimentare e acquisire competenze che potranno essere spese nella pratica professionale. Ma quali sono le riflessioni attorno al futuro professionale mosse dagli studenti in formazione? E come orientare il disegno della propria traiettoria di sviluppo professionale? Queste sono solo alcune delle domande che provano a sintetizzare le sfide che l'orientamento deve affrontare oggi sul piano educativo-formativo. Per gli/le studenti che provano a pensarsi in una prospettiva professionale, definire traiettorie di percorso e di crescita vuol dire "essere pronti per il futuro" e interrogarsi su quale sia il percorso formativo maggiormente in linea con l'idea della professione che vorrebbero svolgere (Parrish, 2016). E ciò potrebbe portare ad incorrere in "errori di percorso" poiché le riflessioni potrebbero basarsi sugli stereotipi che, nell'immaginario collettivo, più caratterizzano quella data professione (Martens, Lacerda, Belfort \& de Freitas, 2016). Il modo in cui viene visto il proprio futuro può fare la differenza e può determinare quindi il perché viene intrapreso un percorso piuttosto che un altro. In tale scenario, il tema del futuro assume un'importanza notevole nel processo educativo insito nell'orientamento poiché da un lato, spinge i singoli in formazione a ripensarsi come maggiormente abili nel fronteggiare i bisogni emergenti; dall'altro, porta a riflettere su nuovi modi di guidare il cambiamento sociale nelle desiderate direzioni future (Facer, 2011). I giovani che si accingono ad intraprendere un percorso universitario che sia in linea con la professione che immaginano di fare, richiama in campo un processo di questioning che è riflessivo, future-oriented e che porta ad esplorare ciò che può essere immaginato del futuro stesso (Burke, 2009). Le scuole e le università devono essere quindi in grado di guidare gli/le studenti nel loro percorso in divenire prevedendo i bisogni emergenti della società, fornendo metodi e promuovendo competenze che possano favorire lo sviluppo di figure professionali capaci di lavorare in contesti in continua evoluzione (Inayatullah, 2006; Stevenson, 2006). Tutto ciò per l'orientamento implica attivare processi di apprendimento che siano in grado di facilitare le prefigurazioni professionali 
a partire dall'analisi di ciò che è conosciuto (ad esempio, abilità, attitudini, desideri professionali) e di ciò che non lo è (il futuro, appunto). I future studies provano a definire maggiormente tale processo di apprendimento prendendo in esame l'anticipatory action learning che è basato sul presente - a partire dalle riflessioni e dalle esperienze pregresse - ed è centrato sull'acquisizione di una conoscenza che è programmata, riprogrammata ed esplorata sulla base dell'ipotetico futuro (Parrish, 2016). La conoscenza che deriva da tale incrocio di prospettive ritrae un futuro che può essere sperimentato $\mathrm{e}$ che permette di esplorare le prospettive di significato utilizzate per prefigurarsi una professione, a partire dalla decostruzione delle potenziali distorsioni (Mezirow, 2003; McDade et al., 2011). L'attivazione del processo di questioning che ne deriva favorisce la riflessione critica sul futuro, inteso quindi come una categoria di analisi basata sulle conoscenze dei partecipanti. Il futuro diventa così una sfida e può essere reinventato. Nell'anticipatory action learning, il processo di questioning segue due fasi principali. La prima riguarda l'esplorazione del futuro, che può essere concepito sulla base delle difficoltà che si possono ipotizzare, delle scelte che si possono perseguire $\mathrm{e}$ delle relazioni che si possono costruire. La seconda fa riferimento alla possibilità di formulare un discorso autoreferenziale, che utilizza il futuro secondo una visione elaborata in forma di metafora, che tiene così conto della soggettività dei partecipanti (Inayatullah, 2005).

Il percorso di ricerca sull'orientamento presentato in questa sede utilizza il futuro come categoria da esplorare all'interno delle epistemologie professionali e parte dal presupposto che il processo di costruzione del percorso professionale non è legato al singolo, ma coinvolge tutte le risorse - scolastiche e universitarie - che lo accompagnano nella costruzione del proprio percorso professionale. I paragrafi che seguono descrivono gli interventi che si collocano nella scia di questo processo di co-costruzione delle prefigurazioni professionali e che hanno coinvolto gli/le studenti del Corso di Laurea in Scienze dell'educazione e della formazione del Dipartimento di Scienze della Formazione, Scienze Umane e della Comunicazione Interculturale dell'Ateneo senese e gli/le studenti delle scuole del territorio provinciale e regionale,

\section{Costruire prefigurazioni professionali all'università. I laboratori di Imprenditoria, genere e leadership femminile}

In questo paragrafo ci concentreremo sui percorsi laboratoriali a sostegno 
dello sviluppo delle prefigurazioni e dell'identità professionale degli/lle studenti all'interno dei corsi di laurea delle 14 università italiane partner del Progetto SUPER-POT. I Corsi di laurea L-19, difatti, sono chiamati a fare i conti con i cambiamenti in atto nelle professionalità emergenti del settore educativo e formativo, impliciti o espliciti che siano. Quali apprendimenti dovrebbe conseguire un educatore alla fine del suo percorso, attraverso quale offerta formativa? Non necessariamente uno studente che si iscrive ai corsi di laurea L-19 ha le idee chiare, spesso ha una serie di rappresentazioni precritiche, frutto di credenze distorte e di mitologie popolari sulle professionalità del mondo educativo (Taylor, 2002; Romano, Bracci, Fabbri, \& Grange, 2018). In questo senso, la ricerca del Progetto SUPER ha perseguito la finalità più ampia di prefigurare le nuove epistemologie professionali che dovranno caratterizzare i laureati di L-19 e i futuri professionisti dell'educazione e della formazione. Che cosa andrò a fare iscrivendomi a questo corso di laurea? Quali esperienze significative di tirocinio, di mobilità estera, di incontri con il mondo del lavoro saranno offerte? Questa università sarà in grado di farmi acquisire un sapere utile per la mia futura pratica professionale? Sono domande che famiglie e studenti si pongono quando cercano informazioni al momento dell'immatricolazione e riguardano la capacità dell'organizzazione universitaria di sostenere la costruzione delle prefigurazioni occupazionali.

Il Corso di laurea in Scienze dell'educazione e della formazione dell'Università di Siena ha sviluppato, nel quadro del Progetto Super, un pattern di azioni sistematiche tra cui laboratori finalizzati a supportare gli/le studenti nell'acquisizione di una cultura del lavoro validata e libera da stereotipi. Il curriculum formale e informale si è arricchito di esperienze laboratoriali in grado di mettere i learner nella condizione di sviluppare competenze metodologiche e trasversali funzionali ad attraversare un mondo lavorativo fatto di situazioni incerte, ambigue e indefinite. Accompagnare gli/le studenti ha significato per il Corso di laurea agevolarne l'incontro con stakeholder locali e organizzazioni educative al fine di:

- creare meticciati con il sapere pratico dei professionisti;

- sostenere l'accesso alla conoscenza pratica tramite esperienze mediate da testimoni privilegiati;

- supportare l'elaborazione critica delle prefigurazioni professionali permettendo a studentesse e studenti di trovare spazi dove poter riflettere sui propri processi di apprendimento (Fabbri \& Romano, 2019).

La scelta di occuparsi delle prefigurazioni professionali non poteva essere un'opzione rimandabile al post-lauream né delegabile solo al Placement, ma si è tradotta, di fatto, nel progettare esperienze di riflessione critica collettiva, 
non individuale, in piccoli e grandi gruppi, ed esperienze di contatto con pratiche esperte (Fabbri, 2018; Fabbri \& Melacarne, 2012). I setting di contaminazione consentono agli studenti di attraversare scenari diversi - quello universitario, quello lavorativo - anticipando problemi, soluzioni, acquisendo competenze pratiche a sostegno dell'occupabilità attraverso un approccio multidimensionale.

Esempi di questi percorsi sono i laboratori a sostegno dell'imprenditorialità e dell'autoimprenditorialità, proposte ibride e meticciate con gli stakeholder del mondo del lavoro, che hanno incluso Call for ideas per start-up imprenditoriali, incontri con testimoni privilegiati all'interno dei laboratori e Hackaton come Innovation Lab. Nello specifico, il Laboratorio di "Imprenditoria, genere e leadership femminile" sviluppato in collaborazione con il Centro Pari Opportunità della Provincia, si è configurato come uno spazio di apprendimento dedicato all'incontro con testimoni provenienti dal tessuto produttivo nazionale, finalizzato alla coltivazione di capacità professionali sfidanti. Il modello concettuale di sfondo è centrato sul research-based learning (Fabbri \& Romano, 2019).

L'articolazione del Laboratorio ha previsto 8 incontri da 3 ore ciascuno con la presenza di 125 partecipanti ripartiti in 5 gruppi da 25 persone ciascuno. La progettazione degli incontri è stata condivisa e negoziata con il Centro per le Pari Opportunità, con i Comitati per l'Imprenditoria Femminile del territorio e con le testimoni privilegiate di volta in volta coinvolte. La Figura 1 sintetizza l'impianto metodologico, i dispositivi didattici e il modello di apprendimento dall'esperienza (Fabbri \& Romano, 2017) che costituiscono i tasselli di un percorso di accompagnamento attivo verso la costruzione di prefigurazioni professionali emergenti.

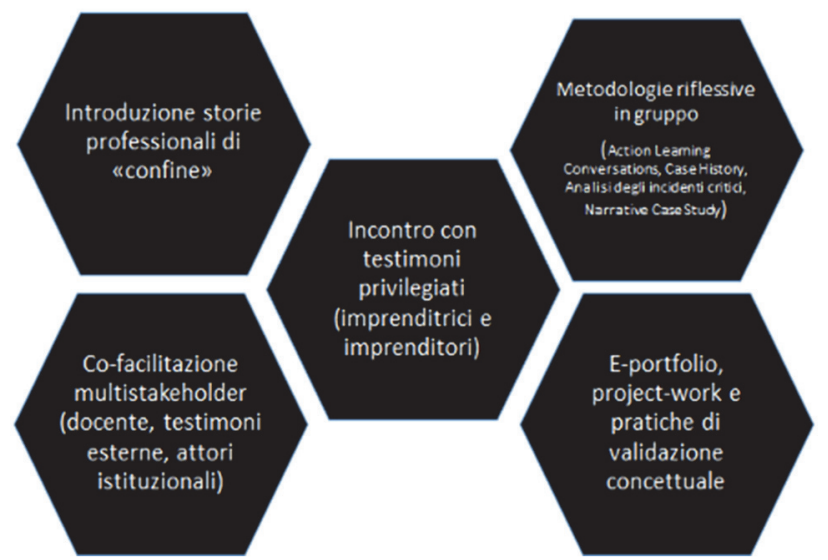

Fig. 1 - Il percorso metodologico del Laboratorio di Imprenditoria, genere e leadership femminile 
Il percorso del Laboratorio ha compreso l'adozione di un set di metodologie finalizzate a supportare processi di professional knowledge sharing e di learning from professional experience:

- introduzione di storie professionali di "confine" e dilemmi professionali disorientanti;

- analisi delle rappresentazioni distorte delle studentesse e degli studenti relative al mondo del lavoro e al loro futuro ruolo professionale;

- predisposizione di incontri con testimoni privilegiati, quali imprenditrici e imprenditori in strutture di co-facilitazione multistakeholder con docenti universitari, attori istituzionali e organizzativi;

- adozione di metodologie riflessive e critico-trasformative, quali analisi degli incidenti critici, case histories e narrative case studies;

- ancoraggi di prospettive critiche, aperte e inclusive a problemi e sfide delle future pratiche professionali;

- acquisizione di schemi di azione e sistemi di intervento e rinforzo attraverso project-work, e-portfolio e pratiche di validazione (Fabbri \& Romano, 2017; Fabbri \& Romano, 2019).

Negli incontri dei laboratori, un primo oggetto di lavoro, più o meno diretto, sono state le immagini interiorizzate e le rappresentazioni di chi fosse e che cosa facesse l'educatore/l'educatrice. A differenza dei medici e degli avvocati, come di altre categorie professionali, la letteratura internazionale concorda nell'evidenziare che educatori e educatrici arrivino all'ingresso nel percorso di studi già con immagini e rappresentazioni consolidate - spesso stereotipate - della figura e del ruolo professionale dell'educatore (Taylor, 2002; Bracci \& Romano, 2017). Hanno già un senso ben sviluppato delle proprie responsabilità e dei ruoli che andranno a svolgere. Pochi si pensano come imprenditori, liberi consulenti e coordinatori di servizi. La rappresentazione più diffusa è quella di un lavoro a contrattualizzazione fissa di tipo dipendente. Quasi nessuno si immagina in un'altra città. L'adozione di metodologie riflessive basate sull'esperienza e per apprendere dall'esperienza (Poell, Yorks, \& Marsick, 2009) hanno facilitato, invece, la costruzione di arene discorsive dove poter validare pre-concezioni e distorsioni e co-costruire schemi di azione più vicini alla pratica professionale.

\section{Professioni da scoprire: i percorsi laboratoriali nella scuola secondaria}

Il percorso d'orientamento laboratoriale sulle prefigurazioni future e professionali realizzato con le scuole, nato nell'ambito del progetto SUPER, ha 
visto coinvolti 5 istituti secondari di secondo grado del territorio aretino (figura 2). La loro partecipazione ha consentito il coinvolgimento di circa 300 studenti delle classi quarte e quinte per cui la riflessione sul futuro professionale è una questione significativa e, come vedremo in alcuni casi, un vero e proprio dilemma disorientante (Mezirow, 2003).

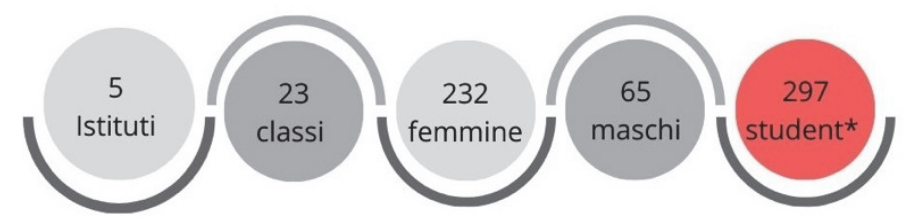

Fig. 2 - Scuole e studenti coinvolti nei laboratori

La metodologia madre con cui sono stati condotti gli incontri è quella laboratoriale. Il Laboratorio è un vero e proprio setting di apprendistato cognitivo in cui si partecipa attivamente, si scopre, si sperimenta, si rielabora (Melacarne, 2011); lavorare in gruppo, far decidere come e in che modo impegnarsi, l'utilizzo del Game-Based Learning si sono rivelati fondamentali per il coinvolgimento degli studenti.

I laboratori sono stati articolati in 3 incontri di due ore ciascuno (tabella 1), e si sono svolti in presenza con gruppi di circa 25 studenti. La loro progettazione è stata condivisa di concerto con i tutor del progetto e con i docenti di riferimento degli istituti coinvolti.

Tab. 1 - Panoramica degli obiettivi e delle metodologie utilizzate negli incontri

\begin{tabular}{|c|c|c|c|}
\hline & Durata & Obiettivi d'apprendimento & Metodologie utilizzate \\
\hline $\begin{array}{l}1^{\circ} \mathrm{In}- \\
\text { contro }\end{array}$ & $2 \mathrm{~h}$ & $\begin{array}{l}\text { - individuare le prefigurazioni pro- } \\
\text { fessionali degli studenti } \\
\text { - sviluppo di conoscenze per vali- } \\
\text { darle } \\
\text { - presentazione del dipartimento e } \\
\text { dei suoi servizi }\end{array}$ & $\begin{array}{l}\text { - Game-Based Learning } \\
\text { - Lego Serious Play } \\
\text { - Jigsaw }\end{array}$ \\
\hline $\begin{array}{l}2^{\circ} \\
\text { Incontro }\end{array}$ & $2 \mathrm{~h}$ & $\begin{array}{l}\text { - approfondimento delle figure pro- } \\
\text { fessionali in uscita dalla classe L-19 } \\
\text { - scoperta dei valori che condizio- } \\
\text { nano e guidano le scelte }\end{array}$ & $\begin{array}{l}\text { - Living Lecture } \\
\text { - Game - Based Learning } \\
\text { - Cooperative Learning }\end{array}$ \\
\hline $\begin{array}{l}3^{\circ} \mathrm{In}- \\
\text { contro }\end{array}$ & $2 \mathrm{~h}$ & $\begin{array}{l}\text { - scoperta dell'importanza della ri- } \\
\text { cerca } \\
\text { - elaborazione del proprio percorso } \\
\text { formativo e professionale }\end{array}$ & $\begin{array}{l}\text { - strumenti di valutazione e } \\
\text { autovalutazione }\end{array}$ \\
\hline
\end{tabular}


Uno degli obiettivi core del percorso di orientamento è stato sfatare le false conoscenze concernenti la professionalità dell'educatore professionale socio-pedagogico. Da un primo approccio con gli studenti è stato chiaro il non riconoscimento della reale declinazione del lavoro dell'educatore accomunato perlopiù alla figura di insegnante, maestro, professore o esclusivamente educatore dei nidi d'infanzia. È stato sorprendente per i partecipanti apprendere l'eterogeneità della professione educativa: attraverso il Jigsaw (Aronson et al., 1978), una metodologia di cooperative learning che facilita e stimola l'apprendimento, incoraggia l'ascolto, l'empatia e il lavoro di squadra, gli/le studenti hanno ricostruito il puzzle della professionalità in oggetto, approfondendo ruoli e caratteristiche dell'educatore di strada, ambientale, penitenziario e anche dell'educatore d'infanzia. L'approfondimento è stato seguito dall'incontro con professionals di questi settori che hanno raccontato la loro esperienza, il loro percorso di vita personale, formativa e lavorativa. Gli/le studenti, in una vera e propria living lecture, hanno così potuto confrontarsi e rapportarsi personalmente con formatori ed educatori provenienti da diversi contesti di lavoro cui hanno posto domande e richiesto specifiche. Le storie ascoltate hanno consentito l'immedesimazione in percorsi di vita altri e il contatto con punti di vista nuovi e sconosciuti. Ad esempio, ascoltare da un educatore penitenziario le difficoltà del suo lavoro non solo in termini di pratica professionale ma anche di attitudine personale è stato particolarmente significativo. Da qui si è registrato un cambio di prospettiva degli studenti circa la comprensione dei setting lavorativi in cui può trovarsi a operare un neo-laureato in L-19 ed è maturata maggiore curiosità verso l'offerta formativa del CdS in Scienze dell'educazione e della formazione.

Altro obiettivo core del percorso è stato il lavoro circa la comprensione delle prefigurazioni professionali dei partecipanti. Alcune delle attività legate a questo obiettivo sono state condotte tramite l'utilizzo di materiali stimolo come i Lego ${ }^{\circledR}$. Agli studenti è stato chiesto di rispondere a domande circa la personale vision del lavoro e il proprio futuro accademico o lavorativo con i materiali forniti e cioè dando corpo e forma ai loro pensieri. Questa tecnica prende il nome di Lego ${ }^{\circledR}$-Serious Play ${ }^{\circledR}$, una metodologia learning by playing (Beltrami, 2017) che facilita la costruzione di strategie d'azione in tempo reale. Significativa è stata la reazione degli studenti a questa proposta: inizialmente vi si erano approcciati quasi con scherno o sfiducia, prendendolo come un "gioco", poi si sono immersi nell'attività e hanno costruito con impegno la loro idea: nel momento in cui è stato chiesto loro di fornire una didascalia all'artefatto creato, sono emerse riflessioni profonde di cui essi stessi erano meravigliati; ciò accade perché nei modelli i partecipanti trasmettono metaforicamente significati profondi che verbalmente difficil- 
mente riescono a rappresentare. Sono così emerse le più disparate rappresentazioni del lavoro: tocchi di laurea per indicare l'intenzione di proseguire il percorso di formazione in università, computer e scrivanie ed edifici ad indicare il desiderio di lavorare in azienda o in un'attività propria, sorrisi rappresentanti il desiderio di lavorare nel sociale, a contatto con le persone, e aerei che indicavano il desiderio di condurre un lavoro all'estero. Accanto ad esse si sono affiancate rappresentazioni meno concrete ma pregne di grandi significati: rappresentazioni di se stessi "perché nel lavoro voglio realizzarmi", un cerchio "che si ripete perché per me l'idea del lavoro è la ripetitività, la monotonia, il fare sempre tutto uguale, a meno che uno riesca a fare carriera", una scala perché "Il lavoro è come la scalata di una montagna: richiede fatica e impegno per essere costruita", un ingranaggio perché "rappresenta la motivazione. Se io faccio un lavoro che mi piace ho un guadagno: io lavoro meglio, sto meglio con la famiglia e meglio con amici e la società".

Partendo dalle conoscenze tacite e confuse degli studenti circa il mondo del lavoro e i desideri più o meno consapevoli, è stato proposto loro il processo ricerca/apprendimento come facilitatore dei processi di comprensione delle proprie prefigurazioni professionali. Tramite il Piano di Sviluppo Professionale, gli/le studenti hanno analizzato i propri desideri delineandoli come obiettivi generali. A questi hanno accostato obiettivi specifici, spacchettando l'obiettivo generale in piccoli step e descrivendo ciò che si intende apprendere individuando le risorse, materiali e umane, e le strategie per farlo. Infine, hanno immaginato le "prove", ossia i segnali che potessero indicare il livello di raggiungimento degli obiettivi specifici. Di seguito l'esempio di un PdS (tabella 2) compilato da una studentessa che ha individuato come obiettivo generale l'iscrizione al CdS in Scienze dell'educazione e della formazione.

Tab. 2 - Esempio di Piano di Sviluppo professionale compilato da una studentessa

\begin{tabular}{|c|c|c|}
\hline Obiettivi specifici & Risorse e strategie & Prove \\
\hline $\begin{array}{l}\text { - Conoscere il piano di } \\
\text { studi } \\
\text { - Conoscere gli sbocchi } \\
\text { professionali } \\
\text { - Sapere se vi è un test di } \\
\text { ammissione } \\
\text { - Comprendere come rag- } \\
\text { giungere la sede delle le- } \\
\text { zioni } \\
\text { - - Capire se ci sono age- } \\
\text { volazioni economiche }\end{array}$ & $\begin{array}{ll}\text { - } & \text { Visitare il sito web } \\
\text { dell'Università } \\
\text { - } & \text { Partecipare all'Open } \\
\text { Day } \\
\text { - }\end{array}$ & $\begin{array}{ll}\text { - } & \text { Stampare il piano di } \\
\text { studi e aver capito quali } \\
\text { esami dovrò sostenere } \\
\text { - } & \text { Aver partecipato alla } \\
\text { giornata di Open Day } \\
\text { - } & \text { Aver raccolto tutte le } \\
\text { informazioni di cui ho } \\
\text { bisogno } \\
\text { - Aver effettuato l'iscri- } \\
\text { zione }\end{array}$ \\
\hline
\end{tabular}


Quella della compilazione del PdS è stata la fase più complessa del ciclo dei laboratori nelle scuole; gli/le studenti apparivano per lo più disorientati di fronte alla richiesta di delineare i propri obiettivi tanto che in diversi hanno lasciato il foglio bianco. Ruolo chiave in questa fase laboratoriale è stato quello giocato dagli studenti tutor universitari, che hanno facilitato la riflessione e rincuorato coloro che non sono riusciti nella prefigurazione, raccontando le loro esperienze e i loro percorsi professionali e di vita in un'azione di peer tutoring. A tal proposito riportiamo la testimonianza di uno studente che al termine del percorso ha dichiarato a proposito del proprio futuro "Ascoltando gli altri ho le idee meno chiare, ma ho capito che la maggior parte dei lavori deve essere inventato ancora e sono fiducioso".

\section{Considerazioni conclusive}

L'implementazione dei laboratori realizzati con gli/le studenti lascia intravedere la presenza di un percorso di transizione tra scuola e università attraverso il quale i soggetti vengono supportati nella definizione delle traiettorie di crescita personale e professionale.

Sostenere la costruzione di prefigurazioni professionali di studenti e studentesse si è tradotto infatti, dal punto di vista metodologico, nella ricerca di pratiche di facilitazione che consentano un guadagno dal punto di vista dell'apprendimento riflessivo. Ad esempio, l'analisi condivisa e collettiva delle storie professionali di "confine" e di "trincea" ha offerto la possibilità di rileggere l'esperienza etero professionale dei testimoni privilegiati attraverso le categorie proprie delle shifting stories (Lanzara, 1990; Fabbri \& Romano, 2019). L'adozione delle metodologie riflessive ha permesso di non cadere nella fascinazione di lavorare per singole competenze richieste, coltivando stili e modelli di leadership considerati dominanti, quanto piuttosto di rapportarsi ad una molteplicità di attori organizzativi per intercettare nuovi schemi di intervento ed azione, preoccupandosi di sviluppare proposte pratiche utili per costruire gli scenari professionali degli studenti.

La metodologia laboratoriale non ha imposto percorsi predefiniti ma ha supportato i learner per ciò che da loro poteva emergere, facilitando il confronto e aprendo riflessioni non solo su se stessi, ma anche su tematiche significative come il tempo, la remunerazione, il genere, la cultura, la disuguaglianza. Una studentessa di una classe quarta di scuola superiore, ad esempio, ha rappresentato il lavoro come una porta da attraversare e una scala da imboccare: "All'inizio il lavoro è un caos e ti senti confusa, c'è poi una scala perché nel corso del tempo è un lavoro progressivo, la scala rappresenta anche una torre perché per le donne può essere più difficile da scalare". 
Come si costruisce allora un percorso di orientamento professionale per studenti in entrata nel mondo del lavoro che sia in grado di sviluppare l'empowerment, accompagnandoli nella definizione degli obiettivi e nella ricerca delle risorse utili a raggiungerli? La sfida a cui scuola, università, istituzioni sono chiamate a rispondere è più che mai attuale e richiede un focus non solo sulle azioni orientanti da progettare, per sostenere gli studenti nella costruzione dei progetti di vita, ma su come progettarli, quali strumenti, approcci e metodologie adottare lavorando per - e con - gli studenti nel difficile compito di co-costruzione delle prefigurazioni professionali. Per farlo è necessario coinvolgere tutte le figure che ruotano attorno allo studente, e costruire un network che allinei scuola e università per realizzare un'azione orientativa che sia significativa ed efficace per tutti gli attori coinvolti.

\section{Riferimenti bibliografici}

Accornero, A. (1999). Perché l'orientamento al lavoro è ancora più importante di ieri. Il Mulino, pp. 132-141.

Aronson, E., Blaney, N., Stephan, C., Sikes, J., \& Snapp, M. (1978). The jigsaw classroom. Beverley Hills.

Beltrami, G. (2017). Lego Serious Play pensare con le mani. Valore per le persone, valore per le organizzazioni. Milano: FrancoAngeli Editore.

Besozzi, E. (1998). Navigare tra formazione e lavoro. Roma: Carocci.

Bianchi, F. (2005). Formare al cambiamento. Roma: Carocci.

Bracci, F., \& Romano, A. (2017). Conceptual metaphors and Apprenticeship Paths as Levers for Professional Development and Learning. In Proceedings of International and Interdisciplinary Conference IMMAGINI? Image and Imagination between Representation, Communication, Education and Psychology, pp. 1-9. Basel, Switzerland: MDPI AG.

Burke, D. (2009). Strategies for using feedback students bring to higher education. Assessment \& Evaluation in Higher Education, 34(1), pp. 41-50.

Da Re, L., Riva, C. (2018). Favorire il successo accademico con il Tutorato Formativo. Scuola democratica, 2, pp. 271-289.

Fabbri, L. (2018). The construction of core contents as a shared scientific practice. Methodologies for transformation of degree program design. Form@re, 18(3), pp. 61-69.

Fabbri, L., \& Melacarne, C. (2012). I giovani e il lavoro. Education Sciences \& Society, 3(1).

Fabbri, L., \& Romano, A. (2017). Metodi per l'apprendimento trasformativo. Roma: Carocci.

Fabbri, L., \& Romano, A. (2019). Costruire infiniti mondi professionali. Prospettive trasformative. Education Sciences \& Society - Open Access, 10(2). Retrieved from https://ojs.francoangeli.it/_ojs/index.php/ess/article/view/8746. 
Facer, K. (2011). Learning futures: Education, technology and social change. Taylor \& Francis.

Inayatullah, S. (2005). Questioning the Future. Tamsui: Tamkang University Press.

Inayatullah, S. (2006). Anticipatory action learning: Theory and practice. Futures, $38(6)$, pp. 656-666.

Lanzara, G. F. (1990). Shifting Stories. Learning from a Reflective Experiment in a Design Process. In: D.A. Shon (Ed.). Reflective practice. A casebook. New York: Teacher College Press.

Martens, C. D. P., Lacerda, F. M., Belfort, A. C., \& de Freitas, H. M. R. (2016). Research on entrepreneurial orientation: current status and future agenda. International Journal of Entrepreneurial Behavior \& Research.

McDade, T. W., Chyu, L., Duncan, G. J., Hoyt, L. T., Doane, L. D., \& Adam, E. K. (2011). Adolescents' expectations for the future predict health behaviors in early adulthood. Social science \& medicine, 73(3), pp. 391-398.

Melacarne, C. (2011). Apprendimento e formazione nella vita quotidiana. Napoli: Liguori Editore.

Messeri, A. (2004). Politiche dell'orientamento e società della conoscenza. Magellano, 19, pp. 39-44.

Mezirow, J. (2003). Apprendimento e trasformazione. Milano: Raffaello Cortina Editore.

MIUR (2014). Linee guida nazionali per l'orientamento permanente, Nota prot. $\mathrm{n}$. 4232.

Moscati, R. (2004), Insegnare dopo la riforma. Il Mulino, 3, pp. 471-478.

Parrish, D. R. (2016). Principles and a model for advancing future-oriented and student-focused teaching and learning. Procedia-Social and Behavioral Sciences, 228, pp. 311-315.

Poell, R. F., Yorks, L., \& Marsick, V. J. (2009). Organizing project-based learning in work contexts: A cross-cultural cross analysis of data from two projects. Adult Education Quarterly, 60(1), pp. 77-93.

Romano, A., Bracci, F., Fabbri, L., \& Grange, T. (2018). Experience-based learning, apprendimento dall'esperienza e sfide femministe. Supportare lo sviluppo dell'identità professionale attraverso pratiche riflessive collettive. Educational Reflective Practices, 1, pp. 9-24.

Rossi, P. (2017). Rapporto con il territorio, orientamento e reclutamento come condizioni abilitanti per una 'buona università', Scuola democratica, 2, pp. 387-401.

Stevenson, T. (2006). From vision into action. Futures, 38(6), pp. 667-672.

Taylor, E. W. (2002). Teaching Beliefs of Graduate Students in Adult Education: A Longitudinal Perspective. In R. M. Cervero, B. C. Courtenay, C. H. Monaghan, (Eds), Comps. The Cyril O. Houle Scholars in Adult \& Continuing Education Program Global Research Perspectives, 2, pp. 120-131. University of Georgia.

Vaira, M. (a cura di) (2007). Dalla scuola all'università. Politiche e pratiche di orientamento in sei paesi europei. Milano: Collana del Cirsis, Led Edizioni. 